\title{
Parler berbère en famille : une revendication identitaire
}

Alexandra Filhon

\section{(2) OpenEdition}

1 Journals

Édition électronique

URL : https://journals.openedition.org/remi/3651

DOI : 10.4000/remi.3651

ISSN : $1777-5418$

Éditeur

Université de Poitiers

\section{Édition imprimée}

Date de publication : 30 juin 2007

Pagination : 95-115

ISBN : 978-2-911627-44-6

ISSN : 0765-0752

Référence électronique

Alexandra Filhon, "Parler berbère en famille : une revendication identitaire », Revue européenne des

migrations internationales [En ligne], vol. $23-n^{\circ} 1$ | 2007, mis en ligne le 30 juin 2010, consulté le 15 avril 2022. URL : http://journals.openedition.org/remi/3651 ; DOI : https://doi.org/10.4000/remi.3651

Ce document a été généré automatiquement le 15 avril 2022.

(c) Université de Poitiers 


\title{
Parler berbère en famille : une revendication identitaire
}

\author{
Alexandra Filhon
}

1 En France, lorsqu'on aborde la question des migrations internationales, on regroupe souvent en un bloc l'immigration dite maghrébine ou nord africaine. Pour qualifier ces populations, on parle des «Arabes », des « arabo-musulmans ». Au mieux on prend en compte le critère de nationalité et l'on perçoit les populations algériennes, marocaines ou tunisiennes à travers leurs appartenances, leurs caractéristiques nationales. La référence aux langues n'est que peu prise en compte, "arabophones » et «berbérophones » ne sont pas distingués les uns des autres ${ }^{1}$.

Or, si actuellement l'arabe est la langue d'immigration la plus parlée en France (Héran, Filhon et Déprez, 2002) le berbère fait également partie des dix langues d'immigration les plus usitées en métropole, ce qui est beaucoup moins connu. Le présent article porte sur une population immigrante mais il s'agit en premier lieu d'un travail sur la transmission familiale. L'objectif est en effet de comprendre comment ces langues arabe et berbère se transmettent d'une génération à une autre et d'un pays à un autre.

Le terme transmission s'inscrit dans le champ de la sociologie de l'éducation et de la socialisation. La transmission d'une langue en tant que "patrimoine culturel " ne s'effectue pas de façon semblable à la transmission d'un "patrimoine immobilier » (Lahire, 1998). Alors que l'héritage matériel passe d'un propriétaire à un autre entraînant en cela une déperdition pour le premier et un enrichissement du second, pour ce qui est du capital culturel celui qui transmet reste toujours en possession de ce qu'il a «transmis » : «Donner à l'autre, c'est l'enrichir sans s'appauvrir » (Lahire, 1998 : 206). De plus, si le patrimoine matériel parvient à se transmettre tout en se gardant inchangé, ce n'est pas le cas du patrimoine immatériel qui se transforme en passant du transmetteur au récepteur. Or, d'après le même auteur "la métaphore de l'“héritage culturel" (ou de la "transmission culturelle") élide les immanquables distorsions, adaptations et réinterprétations que subit le "capital culturel" au cours de sa reconstruction d'une génération à l'autre ou bien d'un adulte à un autre adulte, sous l'effet, d'une part, des écarts entre les supposés "transmetteurs" et les prétendus "récepteurs" et, d'autre 
part, des conditions (des contextes) de cette reconstruction.». Le processus de transmission peut aussi ne pas se finaliser car il ne suffit pas que certaines dispositions soient présentes chez les transmetteurs pour qu'elles parviennent à être incorporées par les récepteurs. La passation du patrimoine immatériel s'inscrit dans la durée contrairement à celle du patrimoine matériel. La transmission peut à la fois mettre du temps à devenir effective mais aussi elle n'est pas nécessairement acquise définitivement et peut évoluer. Enfin dernier point, la transmission d'un capital culturel peut se faire inconsciemment.

Les transmissions familiales sont donc diverses, perceptibles ou non, souhaitées ou pas. Elles peuvent être culturelles, sociales, matérielles, symboliques. C'est pourquoi, il s'agit ici de questionner les rapports intergénérationnels entre parents et enfants mais aussi de montrer que la transmission linguistique familiale ne peut être appréhendée indépendamment des différents contextes de vie. On comparera dans un premier temps les modes de transmission des langues arabe et berbère avant la migration, puis dans un second temps, on observera ce qu'il en est après la migration, une fois les migrants arrivés en France métropolitaine. Avant le départ du Maroc, de la Tunisie ou de l'Algérie, les modes de transmission prennent sens au regard des contextes sociopolitiques de chacun de ces pays, des statuts respectifs de l'arabe et du berbère, enfin des conditions et milieux de vie des populations. En France, pays au sein duquel

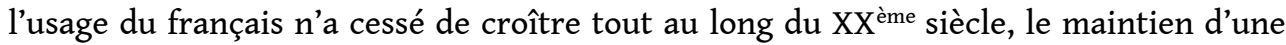
génération à l'autre de ces langues dépend des compétences en français des transmetteurs potentiels mais aussi de l'importance qu'ils accordent à chacune de ses langues. Dans chacun de ces deux contextes nationaux, à deux moments distincts dans la trajectoire des individus, il est impératif de ne pas se limiter à l'étude de la dynamique des relations familiales sans prendre en compte les liens existant entre ces familles et leur environnement social.

On abordera donc dans un premier temps la place du berbère dans le paysage linguistique de l'Afrique du Nord comparée à celle de l'arabe dialectal. Au regard de ce contexte, on s'intéressera plus particulièrement dans un second temps aux modes de transmission de ces langues avant la migration. Enfin on analysera dans un troisième temps les mécanismes de transmission des langues berbères issues du Maroc et d'Algérie après une migration vers la France, dans un contexte linguistique différent.

\section{Méthodologie}

Dans cette recherche ${ }^{2}$, deux types de matériaux ont été exploités. À une enquête quantitative de grande envergure, l'enquête "étude de l'histoire familiale de 1999 ", a été associée la passation d'entretiens biographiques auprès de parent-s migrant-s et de leurs enfants.

7 Jusqu'à présent, la question de la transmission des langues était traitée prioritairement d'un point de vue linguistique à travers l'observation de situations de communication permettant de saisir finement les compétences des individus. La présente étude, sociologique, sur les mécanismes et conditions de transmission des langues arabe et berbère, a été rendue possible par l'existence d'une source de données quantitative, inédite et attendue de longue date: l'enquête "Étude de l'histoire familiale de 1999 " réalisée par l'Insee avec le concours de l'Ined (Lefèvre et Filhon, 2005). Le questionnaire de quatre pages comportait notamment un « volet linguistique » ciblé sur les langues 
familiales, une première dans l'histoire de la statistique publique française (Héran, 2004). Les questionnaires ont été remplis en même temps que les bulletins du recensement par 380000 adultes vivant en métropole, dont les plus âgés sont nés avant la guerre de 1914. Parallèlement à l'exploitation de cette vaste enquête, des entretiens auprès de parents natifs d'Algérie, du Maroc et de Tunisie et de leurs enfants ont été menés. Dans l'ensemble, j'ai reconstitué la trajectoire d'une vingtaine de familles. Lorsque cela a été possible, j'ai rencontré parents et enfants ; mais bien souvent je n'ai pu m'entretenir qu'avec la mère et l'un de ses enfants. Ainsi, dans l'ensemble, de façon plus ou moins formelle, une quarantaine d'entretiens ont été réalisés.

Plusieurs échelles d'observation et plusieurs instruments d'analyse ont donc été utilisés, dans le dessein, non seulement de décrire à grands traits le phénomène étudié, mais aussi de faire émerger plus finement des dynamiques de transmission et de rapport aux différentes langues que sont le français, le berbère et l'arabe dans deux contextes différents : au pays d'origine puis en France.

La distinction que l'on fera nécessairement schématique de la transmission des langues berbère et arabe "avant la migration » et "après la migration » est fondée sur les réponses à deux des questions du questionnaire sur les langues parlées en famille. On demandait au répondant (qui est dans les cas que j'ai retenus une personne originaire du Maroc, d'Algérie ou de Tunisie, ayant migré vers la France et ayant des enfants) : «En quelles langues, dialectes ou patois » leur père, puis leur mère, leur parlaient «d'habitude » vers l'âge de 5 ans. Nous n'avons retenu que les personnes ayant déclaré avoir reçu au moins de l'un de leurs parents la langue arabe ou berbère. Une autre question portait ensuite sur les langues que ces parents, vivant actuellement en métropole, ont eux-mêmes transmises ou retransmises à leurs jeunes enfants, lorsque ceux-ci avaient cinq ans. Cette transmission et retransmission familiale des langues arabe et berbère d'un pays à un autre et d'une génération à une autre est résumée par la figure 1.

Figure 1 : Transmission et retransmission des langues arabe et berbère

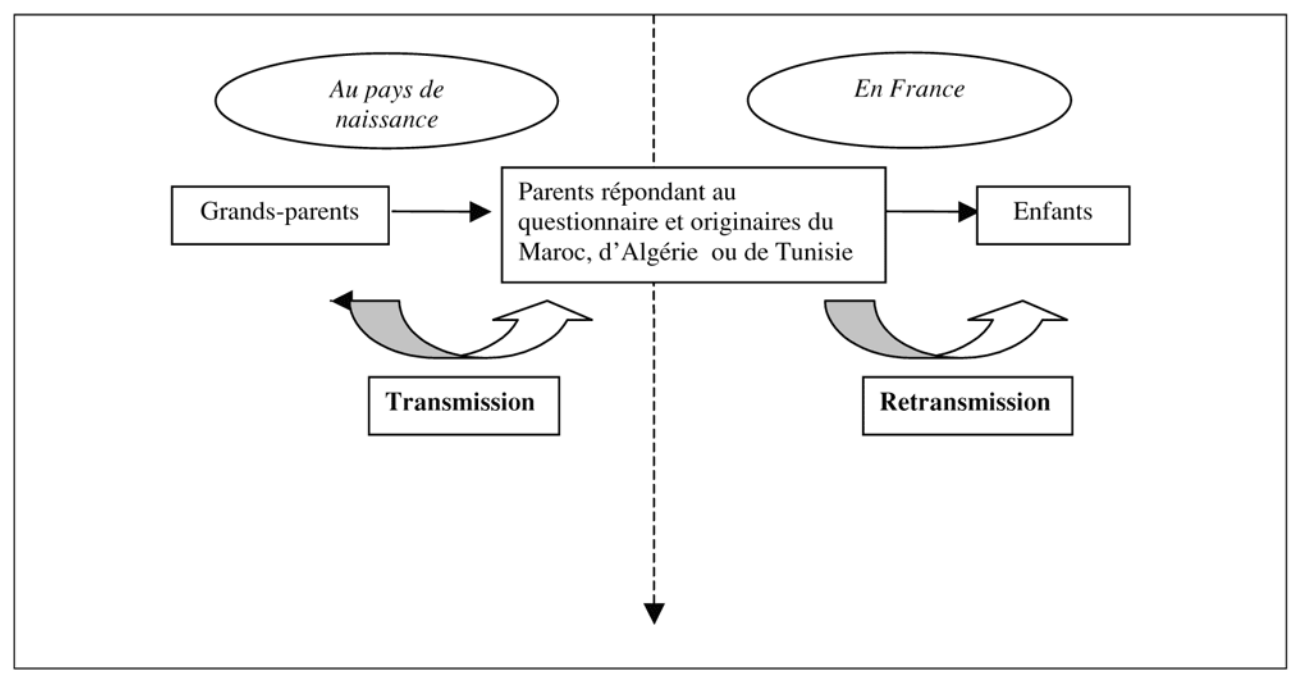




\section{La situation linguistique du berbère en Afrique du Nord}

10 Dans les trois pays d'Afrique du Nord, plusieurs langues se côtoient. On trouve d'un côté des langues apprises dès la naissance et parlées en famille. Il s'agit du berbère et de l'arabe dialectal. Langues orales, le berbère ${ }^{3}$ et l'arabe dialectal regroupent en fait sous ces nominations une diversité de dialectes parlés en Algérie, au Maroc et en Tunisie mais aussi au delà. D'un autre côté, le français et l'arabe classique sont essentiellement des langues d'enseignement et plus rarement des langues familiales en Afrique du Nord. Langues supranationales, ces deux variétés linguistiques ont une forte valeur symbolique bien qu'elles soient peu usitées dans les pratiques quotidiennes.

11 Le mot berbère est dérivé du grec barbaroi et retenu par les Romains dans barbarus, puis récupéré par les Arabes en barbar et enfin par les Français avec berbère. Les populations berbères emploient, elles, le terme Amazigh pour se qualifier et revendiquer leur appartenance commune au delà des frontières nationales; ils nomment leur langue tamazight. Sans revenir à l'aire berbérophone dans les périodes passées, actuellement on trouve trace de cette variété linguistique en Égypte notamment et en Mauritanie, mais également de façon plus visible, au Niger ou encore au Mali avec la présence des Touaregs.

$12 \mathrm{Au}$ Maroc, les populations berbérophones se situent dans les provinces rurales et montagneuses, principalement dans trois zones : au sud et sud-ouest (du Haut Atlas à Souss) il y a le parler tachelhit (chleuh), au centre (Moyen Atlas) le parler tamazight est largement répandu et enfin dans le Nord du pays (Rif), le parler tarifit. En Algérie, les Kabylies situées à l'est d'Alger, densément peuplées pour un territoire de petite envergure et le massif de l'Aurès (parler chaouia) à la frontière tunisienne regroupent la plus grande partie des populations berbères, et les plus actives linguistiquement, c'està-dire celles qui a priori maintiennent le plus fortement l'emploi du berbère. Enfin, des trois pays, la Tunisie est celui qui a été le plus tôt (prise de Carthage par les Arabes en 695) et le plus massivement arabisé. De ce fait, seuls quelques îlots berbérophones subsistent sur l'île de Djerba et dans le sud désertique (S.Chaker, 1999). Ce morcellement des populations berbères explique en partie que cette langue soit restée avant tout un parler vernaculaire ${ }^{4}$ dont il reste peu de traces écrites puisqu'elle demeure en premier lieu une langue de tradition orale.

Réduites toutes deux au rang de dialectes, l'arabe dialectal et le berbère ont toutefois des statuts sociaux inégaux l'une comparée à l'autre. Les arabes tunisien, marocain et algérien sont effectivement nettement plus employés dans les médias, à la télévision et à la radio (Y. Aït Lemkadem, 1999). C'est par exemple le cas pour nombre de téléfilms, spectacles ou pièces de théâtre. Par ailleurs, dans les administrations, les communications se déroulent également en arabe dialectal, si bien que le berbère reste au quotidien la langue la plus dominée socialement.

14 Face à des États qui fondent principalement leur idéologie nationale sur le lien entre arabité et islamité 5 la berbérité dans chacun des pays est peu valorisée mais a tout de même pris de l'importance progressivement. La question se pose essentiellement au Maroc et en Algérie puisque les populations berbérophones en Tunisie sont depuis longtemps quasi-inexistantes. Après l'indépendance de ces pays et l'instauration d'une politique d'unification linguistique au profit de la langue arabe classique, des revendications identitaires des populations berbères ont vu le jour au Maroc et en Algérie. Le " printemps berbère » en 1980 marque notamment une avancée décisive et 
le début d'une volonté de reconnaissance de la langue berbère (tamazight). Ces diverses revendications aboutiront en avril 2002 à l'octroi du statut de langue nationale - mais non officielle contrairement à l'arabe classique - pour le berbère d'Algérie ; au Maroc depuis la rentrée scolaire de 2003, la langue tamazight est enseignée dans de nombreuses écoles primaires.

\section{Profils linguistiques des migrants berbérophones}

15 Cette situation linguistique est essentielle pour comprendre quelles langues sont transmises avant la migration mais aussi après. Globalement, avant la migration la transmission est à $80 \%$ en faveur de l'arabe et à $20 \%$ pour le berbère.

\section{Évolution dans le temps des pratiques linguistiques}

Au-delà du constat que la transmission linguistique familiale est massivement orientée vers l'arabe, il importe d'observer de plus près les configurations linguistiques possibles.

En dehors de la sphère familiale plusieurs langues coexistent et ne sont pas nécessairement cloisonnées les unes par rapport aux autres. Cette diversité linguistique pénètre également dans les foyers mais de façon inégale entre berbérophones et arabophones. En effet, dans l'ensemble moins de deux personnes sur cinq ont déclaré une transmission familiale exclusivement en berbère contre trois personnes sur cinq pour un usage exclusif de l'arabe (tableau 1). Cet écart important rend compte du statut minoré des dialectes berbères. Pour converser avec l'extérieur, c'est-à-dire dans les sphères professionnelles, ou dans le cas de démarches administratives par exemple, les populations berbérophones sont amenées à utiliser l'arabe dialectal, qui au vu des résultats, s'introduit ensuite dans la sphère familiale. Ainsi, ils sont autant à déclarer uniquement une transmission du berbère qu'à déclarer le berbère de pair avec l'arabe. A contrario, parmi les arabophones l'usage du berbère est rare. Enfin, pour les berbérophones comme pour les arabophones, globalement, la langue française est aussi largement présente dans les communications "verticales " parents / enfants, même si comme on le verra par la suite des distinctions importantes s'opèrent d'un pays à un autre. Le français est associé à la pratique du berbère une fois sur trois et il en va de même pour le bilinguisme arabe / français. Là encore, bien que le français soit avant tout une langue apprise à l'école et plus rarement une langue parlée dès la naissance en famille, son emploi de façon plutôt occasionnelle par les parents des futurs migrants à ces derniers atteste également de sa diffusion dans la sphère familiale, peut-être par stratégie d'ascension sociale.

Tableau 1 : Répartition de l'arabe et du berbère, en association ou non avec d'autres langues, par pays de naissance (en \%)

\begin{tabular}{|l|l|l|l||l|}
\hline & Ensemble & Algérie & Maroc & Tunisie \\
\hline $\begin{array}{l}\text { Parmi l'ensemble des arabophones, proportion qui ont reçu... } \\
(\mathrm{N}=4202)\end{array}$ & 100,0 & 100,0 & 100,0 & 100,0 \\
\hline
\end{tabular}




\begin{tabular}{|l|l|l|l|l|}
\hline Parmi eux ont reçu... & & & & \\
\hline •uniquement l'arabe & 57,0 & 49,3 & 65,9 & 58,1 \\
\hline • l'arabe + le berbère & 6,1 & 4,6 & 10,0 & 0,6 \\
\hline • l'arabe + le français & 31,7 & 40,6 & 20,0 & 34,8 \\
\hline • l'arabe + le berbère + le français & 2,1 & 3,4 & 1,4 & 0,3 \\
\hline • autres cas & 3,1 & 2,2 & 2,7 & 6,2 \\
\hline $\begin{array}{l}\text { Parmi l'ensemble des berbérophones, proportions qui ont } \\
\text { reçu... (N = 851) }\end{array}$ & 100 & 100,0 & 100,0 & $/$ \\
\hline Parmi eux ont reçu... & & & & \\
\hline \hline •uniquement le berbère & 37,7 & 41,4 & 29,7 & $/$ \\
\hline \hline • le berbère + l'arabe & 28,5 & 15,1 & 56,1 & \\
\hline \hline • le berbère + le français & 22,8 & 31,6 & 4,8 & \\
\hline • le berbère + l'arabe + le français & 9,9 & 11,1 & 8,0 & $/$ \\
\hline autres cas & 1,1 & 0,8 & 1,4 & $/$ \\
\hline
\end{tabular}

Source : EHF 1999, Insee-Ined.

Champ : Adultes nés au Maroc, en Algérie ou en Tunisie vivant en France au moment de l'enquête, ayant des enfants et qui ont reçu au moins d'un de leurs parents l'arabe et / ou le berbère.

18 Par ailleurs, ces pratiques linguistiques familiales ont évolué au fil du XX ${ }^{\text {ème }}$ siècle (de 1920 à 1975) comme en atteste la figure $2^{6}$. Le monolinguisme arabe ou berbère n'a cessé de diminuer auprès des jeunes générations mais plus ou moins rapidement selon la langue. La pratique exclusive de l'arabe dépassait les $60 \%$ dans les années 1930 à 1950, et la baisse relative de ce monolinguisme ne s'est amorcée qu'au milieu du XX ${ }^{\text {ème }}$ siècle. En revanche, la transmission exclusive du berbère en famille a chuté beaucoup plus rapidement et dès la fin des années trente moins d'une personne sur deux déclarait ne parler que cette langue. Dans l'ensemble, le déclin du monolinguisme et la hausse des pratiques bilingues entre parents et enfants se renforcent au fil du XX ${ }^{\text {ème }}$ siècle en Afrique du Nord. L'arabe et le berbère sont utilisés de plus en plus fréquente en association avec le français. Parmi les générations les plus jeunes près de la moitié des répondants déclarent que leurs parents leur parlaient français en plus de l'arabe ou du berbère. Cette tendance au plurilinguisme est d'autant plus précoce et visible parmi les populations berbérophones qui optent pour un usage du berbère en association avec le français ou l'arabe tandis que les populations arabophones l'associent principalement au français et ce bilinguisme n'a pris le dessus sur le monolinguisme arabe qu'à la fin des années soixante.

19 Ainsi, contrairement à ce que l'on aurait pu penser, c'est principalement après les indépendances que les populations d'Afrique du Nord se sont familiarisées avec la 
langue française. Au sortir de la période coloniale, ces populations sont très majoritairement analphabètes. Les cursus scolaires ont été en partie francisés pendant la colonisation mais finalement peu d'enfants «autochtones " y ont eu accès. À la fin des années cinquante, on estime à environ $85 \%^{7}$ la part de la population analphabète en Afrique du Nord. À peine $15 \%$ des jeunes de 6 à 14 ans étaient scolarisés à la veille des indépendancesrespectives de ces trois pays ${ }^{8}(K a t e b, 2001)$ si bien que peu avaient appris le français par le biais de l'école. Parmi cette minorité scolarisée, moins de $40 \%$ des Marocains se déclarent lettrés en arabe et en français en 1960, contre presque $70 \%$ des Tunisiens d'après le recensement de 1966. Pour chacun de ces trois pays, la massification de l'enseignement va se faire progressivement après l'indépendance en partie sous l'impulsion des politiques d'arabisation.

Figure 2 : Évolution du plurilinguisme ${ }^{9}$ parmi les parents migrants d'Afrique du Nord
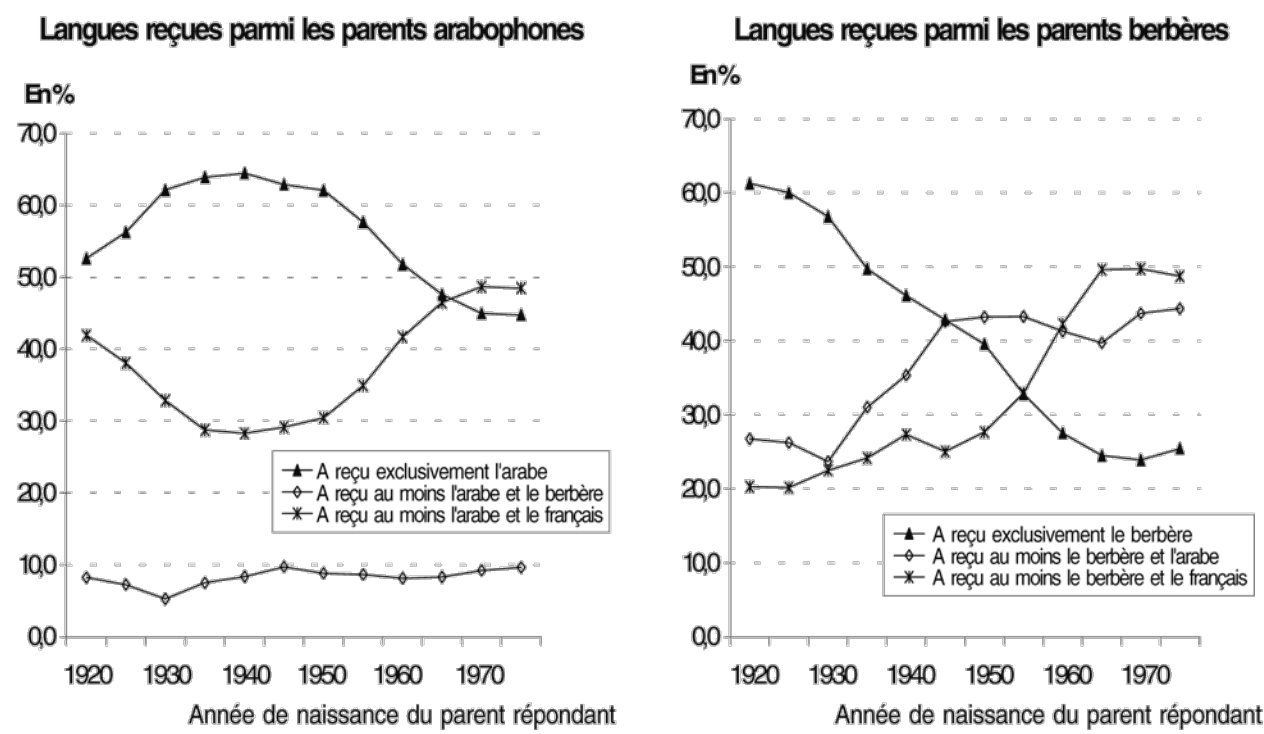

Source : EHF 1999, Ined-Insee.

Champ : Adultes, parents, nés en Afrique du Nord, ayant reçu au moins l'arabe ou le berbère, vivant en métropole. Certains de ces parents figurent sur les deux graphiques s'ils ont déclaré avoir reçu l'arabe et le berbère.

\section{Des modes de transmission distincts entre berbérophones du Maroc et d'Algérie}

Les transmissions linguistiques avant la migration ont évolué avec une prévalence croissante du plurilinguisme. Pour autant, celui-ci prend des formes diverses selon le pays d'origine. En Algérie, les berbérophones reçoivent davantage la langue française alors qu'au Maroc ils associent dans une large mesure le berbère à l'arabe (ci-dessus tableau 1).

Ces tendances distinctes s'expliquent d'une part au regard du contexte socio-historique de chacun de ces pays: plus ou moins rapide massification de la scolarisation des enfants, nature des «liens » tissés avec la France métropolitaine pendant la période coloniale; mais ces profils divergents témoignent aussi d'autre part en Algérie d'une appartenance berbère davantage revendiquée. 
Depuis la fin des années quatre-vingt-dix, les taux de scolarisation des plus jeunes sont massifs en Algérie comme en Tunisie. En revanche, au Maroc plus d'un quart des enfants ne suivent pas d'enseignement (Kateb, 2001). Des écarts persistent également entre les sexes et entre les milieux de vie. D'après le recensement du Maroc de 1994, près des deux tiers des enfants de 8 à 13 ans sont scolarisés, mais moins d'un tiers des jeunes filles vivant en milieu rural (figure 3). On comprend de ce fait pourquoi les berbérophones marocains, issus principalement des milieux ruraux, déclarent dans une très faible proportion avoir reçu aussi le français dans leur enfance. Même pour ceux qui ont été alphabétisés, plus de la moitié de ceux qui vivent en milieu rural indiquent savoir lire et écrire uniquement l'arabe, alors que les citadins, en majorité, se déclarent lettrés en arabe et en français. Comprendre les rapports établis avec la langue française et de quels bagages linguistiques les migrants étaient porteurs lors de leur installation en France est essentiel pour mettre à jour dans un second temps les mécanismes de la transmission des langues natales que sont l'arabe et le berbère. En effet, on peut d'ores et déjà supposer que les migrants non socialisés en langue française avant leur migration ne pourront transmettre à leurs enfants d'autre langue que l'arabe ou le berbère s'ils n'ont eu le temps d'apprendre la langue de leur pays d'installation entre leur arrivée en France et la naissance de leur enfant.

Par le biais de la colonisation et du protectorat, le Maroc la Tunisie et l'Algérie ont tissé des liens "privilégiés » avec la France et notamment avec la langue française. Pour autant c'est surtout depuis les prises d'indépendance que l'emploi du français s'est généralisé, et cela malgré les politiques d'arabisation ${ }^{10}$. Toutefois, la situation vécue par les populations d'Algérie reste particulière. Les cent trente années de colonisation ont nécessairement laissé plus de traces, et les populations, qu'elles soient arabophones ou berbérophones, ont été plus souvent socialisées à la langue française dans leurs familles ou par le biais de relations sociales extérieures. 
Figure 3 : Évolution du niveau d'études des parents migrants berbérophones selon le pays d'origine

Parmi les parents migrants ayant reçu le berbère dans leur enfance

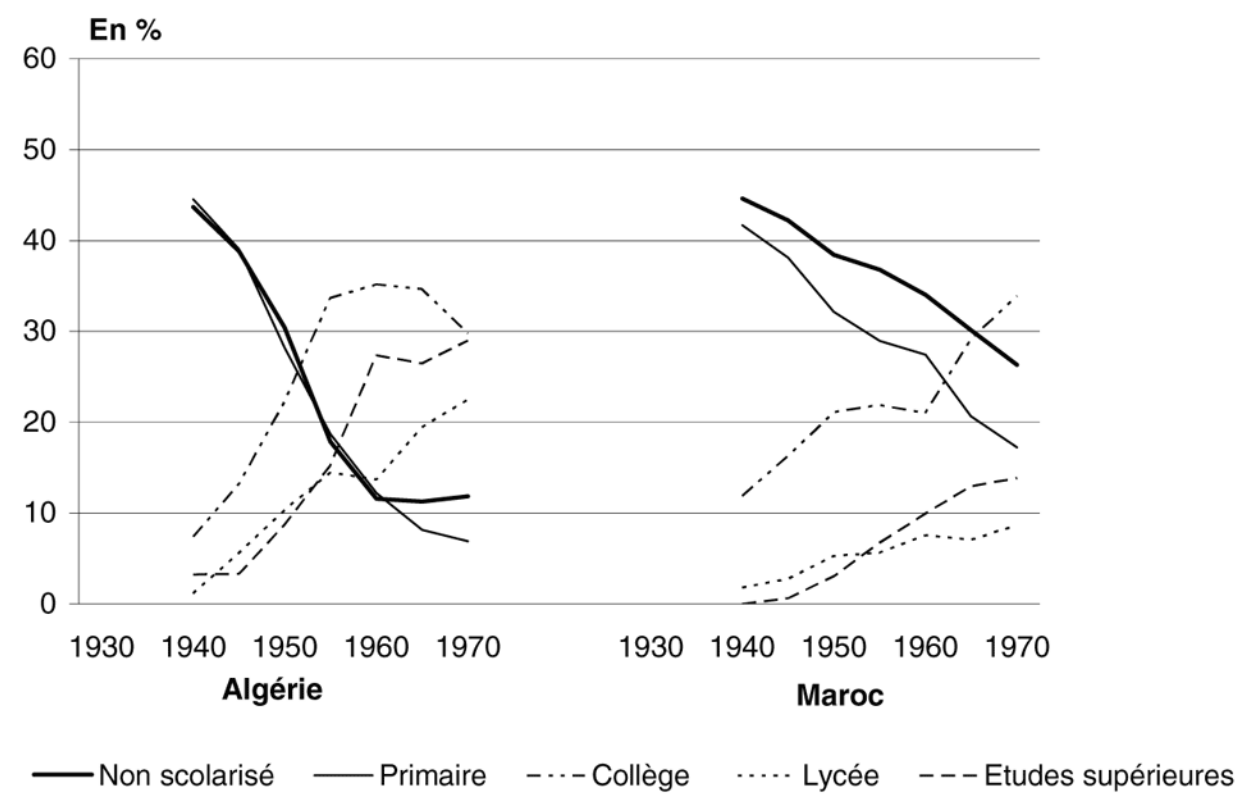

Source : EHF 1999, Ined-Insee.

Champ : Adultes, parents, nés en Algérie ou au Maroc, ayant reçu au moins le berbère dans leur enfance, et vivant en métropole au moment de l'enquête.

Les modes de transmission avant la migration divergent donc d'une langue à l'autre mais aussi d'un pays d'origine à un autre. SI les contextes socio-historiques expliquent en grande partie les écarts de transmission de l'arabe, du berbère et du français, un autre facteur entre également en jeu: une appartenance berbère plus ou moins revendiquée.

Les migrants berbérophones d'Algérie et du Maroc ont un rapport à leur langue natale très différent. Tout d'abord, dans l'enquête Famille de 1999, la façon de nommer la langue berbère n'est pas la même selon les populations. Pour l'Algérie, la mention du «kabyle» est nettement majoritaire alors que les migrants du Maroc déclarent en premier lieu avoir reçu de leurs parents le «berbère ». La nomination «kabyle » fait directement référence à un territoire et à un parler en particulier tandis que déclarer avoir reçu le «berbère " ne témoigne pas d'un attachement territorial. De plus, la visibilité des Kabyles dépasse largement les frontières de l'Algérie. Les berbérophones du Maroc en viennent ainsi eux-mêmes à se déclarer « kabyles » considérant qu'il s'agit de la variété de berbère la plus largement connue; la preuve en est des intitulés fréquemment cités dans l'enquête comme «kabyle marocain" ou "berbère kabyle marocain ».

Cette visibilité du « kabyle » témoigne de la part des populations originaires d'Algérie d'appartenances territoriale et culturelle revendiquées. La moindre valorisation du berbère du Maroc parmi les migrants se perçoit à l'inverse par des nominations telles que « patois » ou « dialecte » que l'on ne retrouve pas en Algérie pour qualifier cette langue.

Enfin, cette valorisation d'une langue et plus largement d'une culture kabyle s'exprime par la proportion importante de ceux qui déclarent n'avoir reçu que cette langue, soit 
plus de 40 \% d'entre eux contre moins de $30 \%$ des berbérophones du Maroc. De même, moins d'un tiers en Algérie ont reçu le berbère de pair avec l'arabe alors que c'est le cas de près des deux tiers de la population berbérophone au Maroc. Mais l'enquête quantitative ne permet pas de savoir si cet usage exclusif d'une langue résulte d'un monolinguisme de fait, ou bien si les parents des migrants, les transmetteurs, ont volontairement parlé uniquement cette langue et non une autre à leurs enfants. Lors des entretiens réalisés en complément de l'enquête quantitative, cette valorisation du kabyle en Algérie comparée au berbère du Maroc s'est vue confirmée. Dans l'extrait d'entretien qui suit ce n'est qu'après environ trois quarts d'heure de conversation que j'apprends que Latifa, mère de quatre enfants, née en 1949 et qui travaille actuellement comme femme de ménage chez des particuliers, est en fait berbère :

Latifa : «Ah moi je suis berbère d'origine, de Ouarzazate, le sud près de Marrakech, je parle couramment, mon mari c'est pareil il est du même coin, on est berbère tous les deux. Moi j'ai grandi à Al Jadida près de Casa, c'est pour ça que je parlais l'arabe, donc ça permet de connaitre le berbère et l'arabe c'est bien. Quand on était jeunes, nos parents ils nous parlaient beaucoup berbère mais après ils nous parlaient arabe aussi, ils parlaient les deux. En principe, les gens dans les villes ils parlent les deux langues par contre les paysans, les montagnards ils parlent que berbère. (...) moi j'aime bien l'arabe, franchement, je le préfere que le berbère, je sais pas, je le sens plus élégant, c'est une langue élégante et la langue du Coran et la langue de beaucoup de choses. L'arabe je trouve elle est plus précieuse que le berbère. "

Cette femme socialisée en famille en langue berbère exprime clairement ici sa préférence pour l'arabe. Cette langue est considérée par cette mère comme prestigieuse alors que le berbère est la langue des ruraux, moins scolarisés. Inversement, parmi les parents originaires de Kabylie, les discours recueillis attestent de l'importance accordée à la langue berbère. C'est le cas par exemple de Malika native d'Algérie, de père berbérophone, et de mère arabophone, qui a peu appris la langue de son père car celui-ci a vécu en France pendant de nombreuses années alors qu'elle résidait à l'époque encore en Algérie. Toutefois, elle percevait une certaine gêne chez son père de savoir que ses enfants n'étaient pas de « vrais » kabyles :

Malika: «[...] du moment où tu rentres en Kabylie sur le territoire kabyle tu n'es plus à Alger, ils ont leur langue ils ont leur administration et progressivement ils se détachent $d u$ pouvoir central.[...] Moi je me sens pas plus kabyle que ça parce que il faut vraiment le vivre $\grave{a}$ fond, je le comprends à peu près on va dire puisque mon père il a jamais voulu parler l'arabe, il parle que le français et le kabyle ça c'est la particularité de tous les Kabyles, ils sont assez... ils se serrent les coudes entre eux. [...] Ma mère elle a fini par apprendre le kabyle (rires) enfin à peu près c'est à se tordre de rire et puis mon père il nous donnait des cours de kabyle, c'était insupportable pour lui que ses enfants ils parlent pas le kabyle. Alors imagine, il nous emmène au bled dans l'arrière pays puisqu'il avait des terres puisqu'il a l'honneur des montagnards, il nous présente à des amis à la famille et les gens très... un peu les montagnards, un peu malsains, ils parlent que le kabyle, ils savent très bien qu'on vient de la capitale qu'on comprend à peu près le kabyle, ils commencent à discuter entre eux tu as compris tant mieux si tu as pas compris tant pis pour toi, ils commencent à se moquer alors nous on a rien pigé, et mon père le soir il est furax (rires) d'ailleurs il nous emmenait plus (rires). »

29 Cet attachement très marqué des Kabyles à leur parler se comprend en partie au regard de la situation sociopolitique du pays. Au Maroc les populations berbères sont attachées à l'arabe pour ce qu'il véhicule et notamment par rapport à l'islam et au roi. En Algérie la volonté centralisatrice des instances au pouvoir est très forte mais en même temps le pays reste davantage "segmenté », et comme le raconte Malika, la Kabylie a longtemps bénéficié d'une grande « autonomie ». 
Les populations berbérophones d'Algérie revendiquent fortement un attachement à leur langue mais parallèlement les Algériens, arabophones et berbérophones, ont aussi été plus familiarisés à la langue française que les populations tunisiennes et marocaines. Avant la migration, les modes de transmission des langues natales berbère et arabe diffèrent d'un pays à un autre compte tenu des politiques d'arabisation instaurées, du rapport entretenu avec la langue française ou encore de la massification plus ou moins rapide de la scolarisation des jeunes générations. Il existe également un rapport subjectif à la langue berbère distinct en Algérie et au Maroc lié en partie à des questions de territoire. Il importe donc à présent de s'interroger sur un maintien éventuel de ces attaches en contexte migratoire.

\section{Quelles transmissions familiales en France?}

Après la migration, près des trois quarts des arabophones ont retransmis leur langue natale à leurs enfants mais seulement environ la moitié des berbérophones (tableau 2). Dans les deux cas, la transmission se fait davantage de façon habituelle - c'est-à-dire régulièrement voire quotidiennement - qu'occasionnellement ${ }^{11}$. Cet apprentissage des langues parentales aux enfants est pour l'arabe comme pour le berbère plus souvent cité par les mères que par les pères et l'écart entre les sexes est plus accentué parmi les berbérophones. Ces deux langues sont donc assez fortement retransmises de la génération des parents migrants à celle de leurs enfants, avec surtout, en premier lieu, un fort maintien de la langue arabe.

Tableau 2 : Retransmission de la langue parentale

\begin{tabular}{|l|l|l|l|l|l|l|}
\hline & \multicolumn{2}{|l|}{$\begin{array}{l}\text { Transmission de l'arabe } \\
\text { (en } \%)\end{array}$} & $\begin{array}{l}\text { Transmission du berbère } \\
\text { (en \%) }\end{array}$ \\
\hline $\begin{array}{l}\text { Parmi les parents socialisés en } \\
\text { arabe ou en berbère... }\end{array}$ & $\begin{array}{l}\text { Ensemble } \\
\mathrm{N}=4202\end{array}$ & $\begin{array}{l}\text { Femmes } \\
\mathrm{N}=2396\end{array}$ & $\begin{array}{l}\text { Hommes } \\
\mathrm{N}=1806\end{array}$ & $\begin{array}{l}\text { Ensemble } \\
\mathrm{N}=851\end{array}$ & $\begin{array}{l}\text { Femmes } \\
\mathrm{N}=447\end{array}$ & $\begin{array}{l}\text { Hommes } \\
\mathrm{N}=404\end{array}$ \\
\hline $\begin{array}{l}\text { Transmission de la langue aux } \\
\text { enfants }\end{array}$ & 71,2 & 74,3 & 68,3 & 54,4 & 60,6 & 49,5 \\
\hline \hline dont transmission habituelle & 44,8 & 46,5 & 43,3 & 34,8 & 37,5 & 32,7 \\
\hline \hline dont transmission occasionnelle & 26,4 & 27,8 & 25,0 & 19,6 & 23,1 & 16,8 \\
\hline
\end{tabular}

Source : EHF 1999, Insee-Ined.

Champ : Parents nés en Algérie, au Maroc ou en Tunisie, ayant reçu au moins l'arabe ou le berbère dans leur enfance et vivant actuellement en France métropolitaine.

\section{Une retransmission fortement associée au français}

Les pratiques linguistiques familiales sont très majoritairement des pratiques de bilinguisme voire plus largement de plurilinguisme. Si les langues arabes et berbères sont assez fortement retransmises d'une génération à l'autre, elles sont aussi très fortement associées au français. Ce résultat atteste à la fois de la "pénétration du 
français » dans les familles et du «maintien de la langue maternelle des parents » dans la sphère privée (Deprez, 1994). À partir d'une enquête menée en région parisienne dans des établissements scolaires auprès de jeunes âgés de 10 à 16 ans, Christine Deprez (1988) a montré que le modèle de communication le plus fréquent est le «parler bilingue " qui se décline selon deux modalités : en alternant les langues ou en les mêlant (cas le plus courant).

Assez logiquement la transmission de la langue française dépend fortement des langues reçues par ces parents. Quel que soit leur pays de naissance, lorsqu'ils ont eux-mêmes entendu parler français dans leur famille, les parents la retransmettent dans de très fortes proportions à leurs enfants et plus des deux cinquièmes d'entre eux de façon exclusive, et d'autant plus fortement lorsqu'il s'agit de pères. Globalement, qu'elles soient berbérophones ou arabophones, les mères transmettent moins souvent que les pères le français de façon exclusive. Elles parlent très majoritairement plusieurs langues à leurs enfants. Enfin, parmi les parents ayant reçu "l'arabe et le berbère » dans leur enfance, les femmes retransmettent au moins le berbère dans la moitié des cas, alors que cette proportion n'est que de deux cinquièmes pour les pères. C'est aussi la seule fois où elles déclarent plus fortement (deux fois plus) que les hommes transmettre de façon exclusive une langue. Les mères ont davantage des pratiques plurilingues avec leurs enfants, excepté dans cette dernière configuration où elles transmettent plus souvent que les hommes uniquement la langue berbère.

\section{Des différences persistantes entre Berbères d'Algérie et du Maroc}

Tout comme c'était déjà le cas avant la migration, la retransmission du berbère en association avec l'arabe après la migration est plus fréquente que la situation inverse. Les statuts divergents de l'arabe et du berbère dans les pays d'origine semblent donc perdurer en France et expliquer cette prévalence d'une langue sur l'autre. De plus, nombre des parents socialisés en berbère dans leur enfance ont également appris à parler en arabe à l'extérieur (ce qui arrive nettement moins souvent dans le cas contraire) et valorisent sa retransmission, en tant que langue du Coran et langue internationale. Ainsi, de ces parents ayant reçu le berbère et l'arabe dans leur enfance, les deux tiers retransmettront au moins l'arabe à leurs enfants, et moins de la moitié au moins le berbère (tableaux 3 et 4 en annexe). Qualifié de "patois" par certains locuteurs, le berbère, bien que largement présent au Maroc et en Algérie, semble plus difficilement retransmis en situation migratoire.

Toutefois, ce constat doit être nuancé pour les Berbères d'Algérie dont notamment les Kabyles qui revendiquent fortement leur origine berbère avant la migration et cherchent à transmettre une partie de cette culture en parlant cette langue à leurs enfants en France. C'est pourquoi le taux de retransmission du berbère en France est supérieur parmi les populations algériennes que parmi celles originaires du Maroc. Les pratiques linguistiques familiales entre les Berbères d'Algérie et les Berbères du Maroc sont clairement distinctes. En plus de la langue berbère, les berbérophones d'Algérie transmettent fortement la langue française et près d'un tiers d'entre eux ne transmettent que cette langue ce qui est loin d'être le cas chez les parents berbérophones du Maroc. En revanche, ces derniers transmettent plus d'une fois sur deux au moins l'arabe à leurs enfants contre seulement $15 \%$ de leurs homologues d'Algérie. 

Kabylie, vont dans ce sens :

"Ma mère elle a été dans une école exclusivement arabe c'est pour ça qu'elle a appris le kabyle et l'arabe, mais sa langue maternelle c'est le kabyle et faut savoir qu'un Kabyle faut pas lui dire "oui t'es Arabe", ça c'est hors de question c'est "non je suis pas Arabe", "pourquoi, t'es Arabe?" "non non j'suis Kabyle...". Enfin, moi quand les personnes elles vont m'aborder et me demander " $t$ 'es de quelle origine ?" le premier réflexe que j'ai c'est de dire "oui je suis Kabyle" et la personne si j'ai un Algérien en face de moi il va le prendre mal il va dire "comment ça t'es Kabyle pourquoi tu dis pas que t'es Arabe?" et je vais dire "non je suis pas Arabe je suis Kabyle si tu veux je te dis que je suis Algérienne c'est vrai mais je suis pas Arabe". Y a une petite nuance qu'il faut cerner, c'est comme si un breton il va vous dire "je suis Parisien" c'est pas possible... Les Kabyles c'est un peuple très nationaliste qui revendique leur identité, ma mère l'arabe elle veut pas le parler il en est hors de question, quand je lui parle parfois en arabe elle me dit "attends on est Kabyle tu me parles en kabyle" ».

Selon leur pays d'origine, les parents berbérophones transmettront dans des proportions significativement différentes les langues arabe, française et berbère. Ces résultats sont dus d'une part à la longue période de colonisation de l'Algérie, colonisation d'autant plus forte dans certaines provinces comme la Kabylie; d'autre part à des politiques d'arabisation dans chacun de ces pays instaurées plus ou moins violemment et aux répercussions assez variables; et enfin à une installation plus ancienne en France des Algériens que des Marocains.

La forte revendication des Kabyles, antérieure à la migration, se maintient a priori en partie en France. La migration entraîne des bouleversements, des recompositions et on aurait pu supposer qu'une telle revendication s'atténue voire disparaisse étant donné que le contexte n'est plus le même. Vu de l'extérieur, on a effectivement tendance à percevoir les arabophones et berbérophones vivant en France comme un même ensemble de migrants. Or les discours des enfants attestent d'un certain maintien de cette visibilité des Kabyles. Les propos de Noria en rendent compte lorsqu'elle raconte l'importance que sa mère accorde à ce que la famille ne soit pas perçue comme "Arabe ». De même, Anisse, jeune étudiant de 18 ans de parents berbères marocains explique cette différence entre Berbères du Maroc et d'Algérie :

« (...) mais je me dis Marocain, je vais dire par rapport... comparé aux Algériens, les Berbères de... les Kabyles se disent... si on leur demande qu'est-ce que vous êtes ils disent Kabyles ils disent pas Algériens, pourtant c'est des Berbères comme nous, moi je me dis Berbère mais avant tout Marocain, un Berbère qui est sur le territoire marocain ça fait partie du Maroc. Les Kabyles ils sont sur le territoire algérien mais c'est comme si c'est pas l'Algérie, elle est là et là y aurait un pays avec la Kabylie, c'est pareil c'est la même chose. Non, nous les Berbères ils se disent Marocains, après on leur demande d'où ils sont et s'il est Berbère il va vous dire je suis d'origine berbère ou d'Agadir. »

Dans le processus de transmission linguistique, le transmetteur potentiel, c'est-à-dire le parent détenteur du capital culturel et social à transmettre, est évidemment un élément clé mais il ne peut être le seul. L'enfant n'est pas seulement un héritier, il est aussi un acteur en partie autonome. Ainsi, quand bien même les parents migrants ont cherché à transmettre l'arabe ou le berbère à leur descendance, et ce dans une très large part comme on a pu le constater, cette transmission du parents vers l'enfant ne peut expliquer à elle seule le rapport aux langues parentales des enfants socialisés en France. La transmission émise, c'est-à-dire concrètement le fait que les parents parlent en famille à leurs enfants l'arabe ou le berbère, ne constitue pas un déterminant suffisant pour appréhender le devenir de ces langues à la génération suivante. Cela signifie, dit autrement, que cette transmission de la part des migrants n'implique pas 
automatiquement que leurs enfants vont eux même parler puis transmettre à leur tour cette ou ces langues à leur propre descendance.

\section{Conclusion} à partir du transmetteur, des caractéristiques sociales et linguistiques dont il est porteur, mais ce processus ne peut se comprendre sans prendre pleinement en considération le récepteur, même si dans cet article le propos a été centré sur le transmetteur. La transmission d'une langue n'est pas un simple patrimoine, un héritage qui pourrait être légué tel quel d'une génération à l'autre. Par ailleurs, cette dialectique parent / enfant doit être réinscrite dans un contexte d'énonciation plus général permettant d'appréhender les conditions de la transmission des langues arabe et berbère avant et après la migration sachant que dans les pays d'origine l'arabe est largement dominant et qu'en contexte migratoire c'est la langue française qui prévaut. Elle occupe le terrain de façon croissante depuis son officialisation au XVI ${ }^{\text {ème }}$ siècle, et plus nettement surtout à partir du XIX ème siècle lorsqu'à travers des politiques linguistiques issues du jacobinisme, le français a clairement été affiché comme la langue de La France, en opposition à tous les autres parlers.

41 Toutefois l'importance des vagues migratoires d'Afrique du Nord vers la France a placé l'arabe et le berbère parmi les principales langues reçues puis retransmises. Les parents arabophones et berbérophones originaires d'Algérie, du Maroc et de Tunisie vivant en France les ont en effet dans une large mesure retransmises à leurs enfants. Cette retransmission se fait très majoritairement de pair avec l'emploi de la langue française (langue à n'en pas douter que tous les enfants scolarisés en France maîtrisent). En tant que principale langue scolaire, professionnelle et administrative, l'apprentissage du français peut être rapide. Les parents, non dépourvus de stratégies, savent de plus que la maitrise de la langue « légitime » assure un pouvoir et une position symbolique sur le «marché linguistique ». Parce qu'elle est la langue des discours et institutions officiels, la langue française produit un effet de distinction au profit des individus qui la détiennent (Bourdieu, 1982). Elle constitue un capital incontournable dans de nombreuses sphères de la vie publique. L'acquisition de cette langue et sa pratique dans la sphère familiale témoignent pour certaines familles d'un sens de l'investissement en vue d'une mobilité sociale.

Avant la migration, la langue arabe a une place prépondérante dans les familles, la langue berbère n'étant transmise que dans un foyer sur cinq. Toutefois, les modes de transmission diffèrent sensiblement en Algérie et au Maroc. Si avant la migration, la revendication d'une appartenance berbère est davantage mise en avant par les populations kabyles et peut se comprendre au regard de l'histoire de ce pays, après la migration cette distinction persiste. En effet, les modes de retransmission suite à la migration rendent compte d'une part d'un maintien significatif du berbère en France, et d'autre part de retransmissions distinctes entre Algériens et Marocains. En France, les parents berbérophones natifs d'Algérie transmettent leur langue natale et le français assez fortement alors que pour les migrants originaires du Maroc, la langue berbère est légèrement moins retransmise car un certain nombre de parents optent pour un bilinguisme franco-arabe.

Revue européenne des migrations internationales, vol. 23 - $n^{\circ} 1$ | 2007 

leurs origines nationales ou leurs langues natales.

\section{BIBLIOGRAPHIE}

ABELLA Manolo I. (1990) “Sex Selectivity of Migration Regulations Governing International Migration in Southern and South-Eastern Asia" in International Migration Policies and the Status of Female Migrants, Proceedings of the United Nations Expert Group Meeting on International Migration Policies and the Status of Female Migrants, San Miniato, Italy, 28-31 March, pp. 241-252.

AÏT LEMKADEM Youssef (1999) « Le Berbère dans le paysage linguistique et culturel marocain », in Francis Manzano dir. Langues du Maghreb et du Sud méditerranéen, Rennes, Presses universitaires de Rennes, pp. 25-36.

BENRABAH Mohamed (1999) Langue et pouvoir en Algérie : histoire d'un traumatisme linguistique, Paris, Éditions Séguier, 348 p.

BENRABAH Mohamed (2002) École et plurilinguisme en Algérie : un exemple de politique linguistique éducative « négative », Éducation et Société Plurilingues, n 13, décembre, pp. 73-80.

BOUKOUS Ahmed (1996) La politique linguistique au Maroc : enjeux et ambivalences, in Juillard Caroline et Calvet Lous-Jean dir, Les politiques linguistiques, mythes et réalités, éd. AUPELF-UREF, pp. 73-82.

BOURDIEU Pierre (1982) Ce que parler veut dire : l'économie des échanges linguistiques, Paris : Fayard, $243 \mathrm{p}$.

BOUZIRI Raja (1999) La variation des pratiques langagières des jeunes d'origine maghrébine à la Goutte d'Or, thèse de doctorat en sociolinguistique, Paris, EHESS.

CAMPS Gabriel (1980) Berbères. Aux marges de l'Histoire, Toulouse, Éditions des Héspérides, 352 p. [Réédité sous le titre : Berbères. Mémoire et identité, Paris, Éditions Errances, 1987.]

CASSAN Francine, HÉRAN François, TOULÉMON Laurent (2000) « Étude de l'histoire familiale : l'édition 1999 de l'enquête Famille », Courrier des Statistiques, n 93, mars, pp. 25-37.

CHAKER Salem (1999) La langue berbère : entre marginalisation et affirmation, in Manzano Francis Langues du Maghreb et du Sud méditerranéen, Cahiers de Sociolinguistique, $\mathrm{n}^{\circ} 4$, Presses Universitaires de Rennes, pp. 11-24.

DÉPREZ Christine (1994), Les enfants bilingues : langues et familles, Paris, Didier, CREDIF, 207 p.

FILHON Alexandra (2004) Transmission familiale des langues arabe et berbère en France : langues, cultures et identités des migrants nord-africains et de leurs enfants, Thèse de Sociologie sous la dir. de Catherine Rollet, Université de Versailles Saint-Quentin-en-Yvelines.

GALLISSOT René (2000) Le Maghreb de traverse, Paris, Éditions Bouchene, 145 p.

GRANDGUILLAUME Gilbert (1983) Arabisation et politique linguistique au Maghreb, Paris, Maisonneuve et Larose, $210 \mathrm{p}$. 
HÉRAN François, FILHON Alexandra, DEPREZ Christine (2002) La dynamique des langues en France au fil du XXème siècle, Population et Sociétés, $n^{\circ} 376$, février, 4 p.

HÉRAN François (2004) Une approche quantitative de l'intégration linguistique en France, Hommes et Migrations, $\mathrm{n}^{\circ}$ 1252, novembre-décembre 2004, pp. 10-24.

KATEB Kamel (2001) Européens, « indigènes » et juifs en Algérie : 1830-1962 : représentations et réalités des populations, Paris, Presses universitaires de France, Institut national d'études démographiques, $386 \mathrm{p}$.

LACOSTE Camille et Yves dir. (1995) Maghreb. Peuples et civilisations, Paris, La Découverte 208 p.

LAHIRE Bernard (1998) L'homme pluriel, les ressorts de l'action, Paris, Nathan, $392 \mathrm{p}$.

LAROUSSI Fouad (dir.) (1997) Plurilinguisme et identités au Maghreb, Rouen, Publications de l'Université de Rouen, $124 \mathrm{p}$.

LEFÈVRE Cécile et FILHON Alexandra dir. (2005) Histoires de familles, histoires familiales : les résultats de l'enquête Famille de 1999, Ined, Les Cahiers de l'Ined n 156, 641 p.

MOATASSIME Ahmed (1992) Arabisation et langue française au Maghreb, Paris, IEDES Coll. Tiers Monde, PUF, $176 \mathrm{p}$.

SAKKOUNI Ahmed (1997) Le contexte sociolinguistique des familles immigrées : l'exemple de familles marocaines, Migrations Santé, $n^{\circ}$ 92, pp. 9-26.

SIMON Jacques (2000) L'immigration algérienne en France. Des origines à l'indépendance, Paris, Éditions Paris-Méditerranée, Coll. Documents, témoignages et divers, $412 \mathrm{p}$.

SIMON Patrick (1997) L'acculturation linguistique. Utilisation du français et transmission de la langue des immigrés à leurs enfants, Migrants-Formation, n 108, mars, pp. 53-66.

VARRO Gabrielle (2003) Sociologie de la Mixité. De la mixité amoureuse aux mixités sociales et culturelles, Paris, Belin, 253 p.

ZEHRAOUI Ahsène dir. (1999) Familles d'origine algérienne en France. Étude sociologique des processus d'intégration, Paris, L'Harmattan, $320 \mathrm{p}$.

\section{ANNEXES}


Tableau 3 : Quelle retransmission de l'arabe selon les langues reçues, le sexe et le pays de naissance?

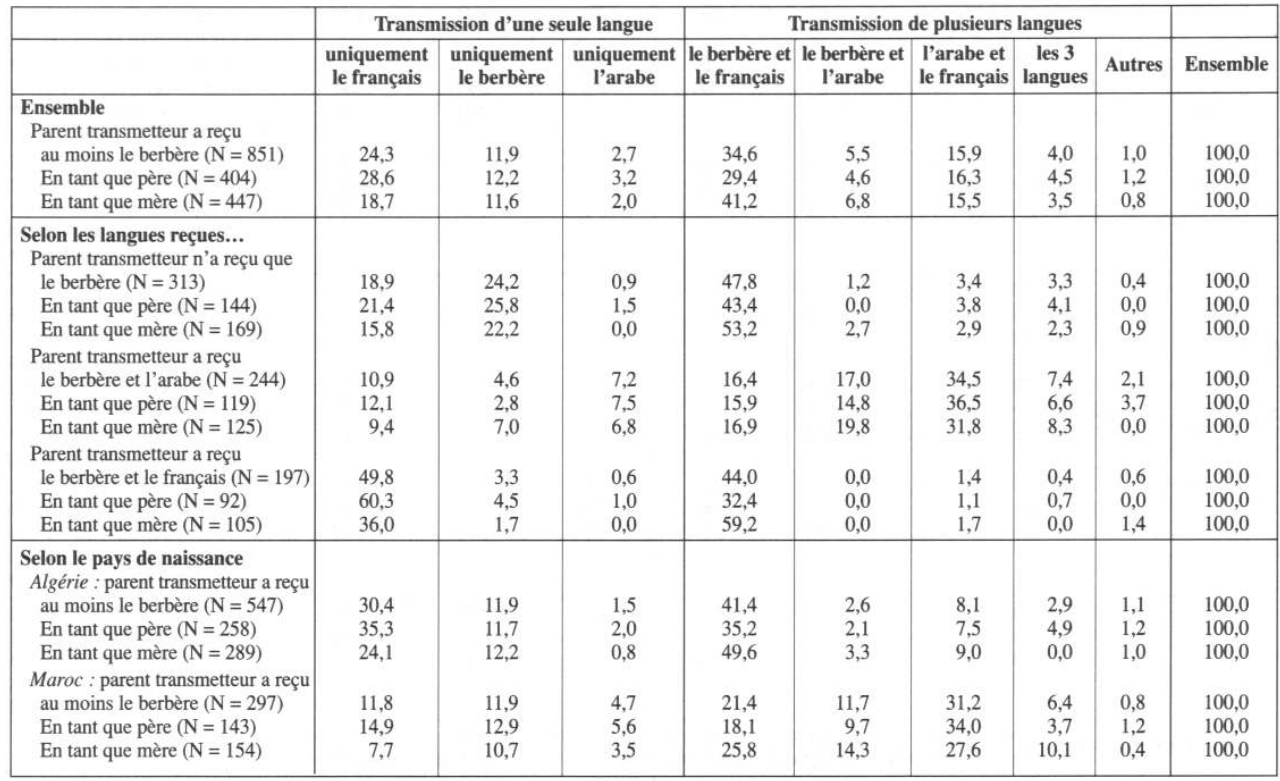

Source : EHF 1999, Ined-Insee.

Tableau 4 : Quelle retransmission du berbère selon les langues reçues, le sexe du parent et le pays de naissance?

\begin{tabular}{|c|c|c|c|c|c|c|c|c|c|}
\hline & \multicolumn{3}{|c|}{ Transmission d'une seule langue } & \multicolumn{5}{|c|}{ Transmission de plusieurs langues } & \multirow[b]{2}{*}{ Ensemble } \\
\hline & $\begin{array}{l}\text { uniquement } \\
\text { le français }\end{array}$ & $\begin{array}{c}\text { uniquement } \\
\text { l'arabe }\end{array}$ & $\begin{array}{c}\text { uniquement } \\
\text { le berbère }\end{array}$ & $\begin{array}{l}\text { l'arabe et } \\
\text { le français }\end{array}$ & $\begin{array}{l}\text { l'arabe et } \\
\text { le berbère }\end{array}$ & \begin{tabular}{|c|} 
le berbère et \\
le français
\end{tabular} & \begin{tabular}{|c|} 
les 3 \\
langues
\end{tabular} & Autres & \\
\hline $\begin{array}{l}\text { Ensemble } \\
\text { Parent transmetteur a reçu } \\
\text { au moins l'arabe }(N=4202) \\
\text { En tant que père }(N=1806) \\
\text { En tant que mère }(N=2396)\end{array}$ & $\begin{array}{l}22,6 \\
25,4 \\
19,7 \\
\end{array}$ & $\begin{array}{l}15,5 \\
16,5 \\
14,4 \\
\end{array}$ & $\begin{array}{l}0,4 \\
0,4 \\
0,5 \\
\end{array}$ & $\begin{array}{l}56,6 \\
53,0 \\
60,5 \\
\end{array}$ & $\begin{array}{l}1,1 \\
1,1 \\
1,1 \\
\end{array}$ & $\begin{array}{l}1,6 \\
1,5 \\
1,7 \\
\end{array}$ & $\begin{array}{l}0,6 \\
0,7 \\
0,5 \\
\end{array}$ & $\begin{array}{l}1,6 \\
1,5 \\
1,6 \\
\end{array}$ & $\begin{array}{l}100,0 \\
100,0 \\
100,0\end{array}$ \\
\hline $\begin{array}{l}\text { Selon les langues reçues... } \\
\text { Parent transmetteur n'a reçu que } \\
\text { l'arabe }(\mathrm{N}=2366) \\
\text { En tant que père }(\mathrm{N}=1064) \\
\text { En tant que mère }(\mathrm{N}=1302)\end{array}$ & $\begin{array}{l}13,0 \\
15,1 \\
10,4\end{array}$ & $\begin{array}{l}24,2 \\
24,2 \\
24,1\end{array}$ & $\begin{array}{l}0,1 \\
0,1 \\
0,0\end{array}$ & $\begin{array}{l}62,2 \\
59,8 \\
65,1\end{array}$ & $\begin{array}{l}0,0 \\
0,0 \\
0,0\end{array}$ & $\begin{array}{l}0,1 \\
0,3 \\
0,0\end{array}$ & $\begin{array}{l}0,0 \\
0,0 \\
0,0\end{array}$ & $\begin{array}{l}0,4 \\
0,5 \\
0,4\end{array}$ & $\begin{array}{l}100,0 \\
100,0 \\
100,0\end{array}$ \\
\hline $\begin{array}{l}\text { Parent transmetteur a reçu } \\
\text { l'arabe et le berbère }(\mathrm{N}=244) \\
\text { En tant que père }(\mathrm{N}=119) \\
\text { En tant que mère }(\mathrm{N}=125)\end{array}$ & $\begin{array}{r}10,9 \\
12,1 \\
9,4\end{array}$ & $\begin{array}{l}7,2 \\
7,5 \\
6,8\end{array}$ & $\begin{array}{l}4,6 \\
2,8 \\
7,0\end{array}$ & $\begin{array}{l}34,5 \\
36,5 \\
31,8\end{array}$ & $\begin{array}{l}17,0 \\
14,8 \\
19,8\end{array}$ & $\begin{array}{l}16,4 \\
15,9 \\
16,9\end{array}$ & $\begin{array}{l}7,4 \\
6,6 \\
8,3\end{array}$ & $\begin{array}{l}2,1 \\
3,7 \\
0,0\end{array}$ & $\begin{array}{l}100,0 \\
100,0 \\
100,0\end{array}$ \\
\hline $\begin{array}{l}\text { Parent transmetteur a reçu } \\
\text { l'arabe et le français }(N=1384) \\
\text { En tant que père }(N=519) \\
\text { En tant que mère }(N=865)\end{array}$ & $\begin{array}{l}40,1 \\
47,5 \\
33,9\end{array}$ & $\begin{array}{l}3,5 \\
5,1 \\
2,1\end{array}$ & $\begin{array}{l}0,1 \\
0,0 \\
0,2\end{array}$ & $\begin{array}{l}54,1 \\
46,4 \\
60,5\end{array}$ & $\begin{array}{l}0,0 \\
0,0 \\
0,0\end{array}$ & $\begin{array}{l}0,3 \\
0,0 \\
0,5\end{array}$ & $\begin{array}{l}0,0 \\
0,0 \\
0,0\end{array}$ & $\begin{array}{l}1,9 \\
1,0 \\
2,7\end{array}$ & $\begin{array}{l}100,0 \\
100,0 \\
100,0\end{array}$ \\
\hline $\begin{array}{l}\text { Selon le pays de naissance } \\
\text { Algérie: parent transmetteur a reçu } \\
\text { au moins l'arabe }(\mathrm{N}=1987) \\
\text { En tant que père }(\mathrm{N}=839) \\
\text { En tant que mère }(\mathrm{N}=1148)\end{array}$ & $\begin{array}{l}26,8 \\
29,0 \\
24,4\end{array}$ & $\begin{array}{l}13,9 \\
16,4 \\
11,2\end{array}$ & $\begin{array}{l}0,6 \\
0,4 \\
0,8\end{array}$ & $\begin{array}{l}54,0 \\
49,8 \\
58,6\end{array}$ & $\begin{array}{l}0,8 \\
0,7 \\
0,9\end{array}$ & $\begin{array}{l}2,0 \\
1,5 \\
2,5\end{array}$ & $\begin{array}{l}0,5 \\
0,9 \\
0,0\end{array}$ & $\begin{array}{l}1,4 \\
1,3 \\
1,6\end{array}$ & $\begin{array}{l}100,0 \\
100,0 \\
100,0\end{array}$ \\
\hline $\begin{array}{l}\text { Maroc: parent transmetteur a reçu } \\
\text { au moins l'arabe }(\mathrm{N}=1672) \\
\text { En tant que père }(\mathrm{N}=740) \\
\text { En tant que mère }(\mathrm{N}=932)\end{array}$ & $\begin{array}{l}16,4 \\
18,0 \\
14,7\end{array}$ & $\begin{array}{l}18,8 \\
18,9 \\
18,8\end{array}$ & $\begin{array}{l}0,4 \\
0,4 \\
0,4\end{array}$ & $\begin{array}{l}58,2 \\
56,5 \\
60,2\end{array}$ & $\begin{array}{l}1,8 \\
1,8 \\
1,8\end{array}$ & $\begin{array}{l}1,7 \\
2,1 \\
1,2\end{array}$ & $\begin{array}{l}0,9 \\
0,6 \\
1,3\end{array}$ & $\begin{array}{l}1,6 \\
1,7 \\
1,6\end{array}$ & $\begin{array}{l}100,0 \\
100,0 \\
100,0\end{array}$ \\
\hline $\begin{array}{l}\text { Tunisie : parent transmetteur a reçu } \\
\text { au moins l'arabe }(\mathrm{N}=543) \\
\text { En tant que père }(\mathrm{N}=227) \\
\text { En tant que mère }(\mathrm{N}=316)\end{array}$ & $\begin{array}{l}26,2 \\
34,9 \\
17,6\end{array}$ & $\begin{array}{r}11,4 \\
9,8 \\
13,0\end{array}$ & $\begin{array}{l}0,0 \\
0,0 \\
0,0\end{array}$ & $\begin{array}{l}60,5 \\
53,3 \\
67,5\end{array}$ & $\begin{array}{l}0,0 \\
0,0 \\
0,0\end{array}$ & $\begin{array}{l}0,0 \\
0,0 \\
0,0\end{array}$ & $\begin{array}{l}0,0 \\
0,0 \\
0,0\end{array}$ & $\begin{array}{l}2,0 \\
2,0 \\
1,9\end{array}$ & $\begin{array}{l}100,0 \\
100,0 \\
100,0\end{array}$ \\
\hline
\end{tabular}

Source : EHF 1999, Ined-Insee. 


\section{NOTES}

1. Je remercie Alain Blum pour la lecture attentive de cet article.

2. Elle est le fruit de cinq années de travail de thèse (Filhon, 2004).

3. La langue berbère possède pourtant depuis plus de deux millénaires un système d'écriture appelé tifinagh, et plus récemment certains écrivains ou poètes utilisent l'alphabet latin ou arabe.

4. Le parler « vernaculaire " sert à la communication d'une aire ou d'un groupe. Par opposition, un parler "véhiculaire » renvoie davantage à la communication supra-locale ou entre des groupes de langue différente.

5. La domination de l'arabe sur le berbère s'explique notamment par le lien qui existe entre l'arabe classique et l'Islam. En tant que langue sacrée permettant la lecture du Coran, elle acquiert une forte valeur symbolique qui a notamment contribué à sa diffusion et son maintien au fil des siècles.

6. Précisons que les débuts des représentations graphiques peuvent être quelque peu imprécises et biaisées. Il s'agit en effet de migrants nés dans les années 1920-1930, âgés de plus de 70 ans, à qui l'on demande de se remémorer des souvenirs très lointains. De plus, parmi les migrants de cette tranche d'âge, ceux qui ont vécu dans les situations les plus précaires sont décédés plus jeunes et donc seront moins représentés; enfin, un certain nombre d'entre eux sont également retournés vivre dans leur pays de naissance après leur retraite. À l'inverse, pour les dernières générations nées dans les années soixante-dix et déjà en France en 1999, on peut supposer que certains sont arrivés enfants ou adolescents et qu'ils ont donc entendu parler français assez tôt.

7. Tunisie : $84,7 \%$ de la population d'après le recensement de 1956, Maroc: $87 \%$ d'après le recensement de 1960, Algérie : $91 \%$ en 1954.

8. Rappelons que l'établissement des régimes de protectorats en Tunisie et au Maroc datent respectivement de 1881 et 1912 et ont pris fin en 1956. Quant à l'Algérie, elle a été colonisée par la France durant plus de 130 années : de 1830 à 1962.

9. Le plurilinguisme est le fait de comprendre et / ou parler plusieurs langues, quelles que soient leur nombre. Dans la figure 2, les répondants ayant reçu « au moins l'arabe et le français » ou " au moins l'arabe et le berbère » peuvent avoir déclaré plus de deux variétés linguistiques. Le bilinguisme, un type de plurilinguisme, est précisément le fait de comprendre et / ou de parler deux langues.

10. Pour en savoir plus sur la mise en place des politiques d'arabisation en Algérie, au Maroc et en Tunisie, lire les travaux de Gilbert Granguillaume et notamment son ouvrage de 1983 : Arabisation et politique linguistique au Maghreb, publié à Maisonneuve et Larose. Plus précisément sur la question algérienne, lire les travaux de Mohamed Benrabah dont son ouvrage de 1999 intitulé Langue et pouvoir en Algérie: histoire d'un traumatisme linguistique etpublié aux éditions Séguier.

11. Dans le volet linguistique de l'enquête Famille, deux lignes de réponse permettaient aux répondants d'indiquer les variétés linguistiques transmises soit "d'habitude " pour la première ligne, soit «aussi» pour la seconde ligne, que l'on appelle par convention transmission «occasionnelle». Sur chacune de ces lignes plusieurs langues pouvaient être citées ce qui signifie que la hiérarchisation des langues par les répondants n'était pas automatique. Ils ont pu pour certains choisir d'indiquer plusieurs langues sur la première ligne et aucune sur la seconde ligne. 


\section{RÉSUMÉS}

Dans les pays d'origine, au Maroc, en Tunisie et en Algérie, la transmission de l'arabe et du berbère diffère selon les contextes sociopolitiques, les statuts respectifs de ces langues, enfin les conditions et milieux de vie des populations. Avant la migration, l'usage de l'arabe a une place prédominante dans les familles, la langue berbère n'étant parlée que dans un foyer sur cinq. Toutefois, la fréquence de sa transmission varie sensiblement entre l'Algérie et le Maroc. En France, l'usage du français n'a cessé de croître tout au long du XX ${ }^{\text {ème }}$ siècle et prévaut désormais. Le maintien de l'arabe et du berbère dépend des compétences en français des migrants et de leurs rapports à leur-s langue-s natale-s. La revendication d'une appartenance berbère est davantage mise en avant par les populations kabyles qui transmettent plus fréquemment que les berbères marocains leur langue à leurs enfants.

To speak Berber inside the Family: claiming an Identity. In the countries of origin, in Morocco, in Tunisia and in Algeria, the transmission of Arabic and Berber makes sense in the socio-political context of these countries, according to the statute of each of these languages, and also to the conditions and ways of life of the populations. Before the migration, the use of Arabic plays a predominant part in families, the Berber language being spoken only in one home out of five. However, between Algeria and Morocco, the transmission of the Berber language varies appreciably. In France, the practices of French did not stop growing throughout the 20th century and prevails henceforth. However the Arabic and Berber languages are two of the ten main foreign linguistics varieties. After the migration, the preservation of these languages depends on how skilled in French the migrants are and on their relations to their native languages. The demand of a Berber belonging is, for example, put forward more by kabyle populations than by Moroccan Berbers.

Hablar berebere en familia : una reivindicación de identidad. En los países de origen, Marruecos, Túnez y Argelia, la transmisión del árabe y del berebere difiere en función de los contextos sociopolíticos, de los estatutos respectivos de estas lenguas y de las condiciones de vida de las poblaciones. Previamente a la migración, el uso del árabe goza de una posición predominante en las familias mientras que la lengua berebere se habla únicamente en un hogar de cada cinco. Sin embargo, la frecuencia de su transmisión varía sensiblemente entre Argelia y Marruecos. En Francia, el uso del francés no ha cesado de progresar a lo largo del siglo XX y, en estos momentos, prevalece. Que el árabe y el berebere se mantengan depende del nivel de francés de los inmigrantes y de los lazos con su/s lengua/s natal/es. La reivindicación de la identidad berebere es así mayor entre las poblaciones kavillas quienes transmiten la lengua a sus hijos con más frecuencia que los berebere marroquíes.

\section{INDEX}

Index géographique : Algérie, Maroc, Tunisie

Mots-clés : appartenance, berbère, identité culturelle, identité ethnique, langue d'origine 
AUTEUR

ALEXANDRA FILHON

Maître de Conférences, Laboratoire Printemps, CNRS - Université Paris X, 200 avenue de la

République, 92001 Nanterre cedex, France. Courriel : filhon@ined.fr 